K. Tojo

Nagoya Math. J.

Vol. 143 (1996), 31-57

\title{
COVARIANT DERIVATIVES ON KÄHLER C-SPACES
}

\author{
KOJI TOJO
}

\section{Introduction}

Let $(M, g)$ be a Kähler $C$-space. $R$ and $\nabla$ denote the curvature tensor and the Levi-Civita connection of $(M, g)$, respectively.

In [6], Takagi have proved that there exists an integer $n$ such that

$$
\hat{\nabla}^{n-1} R \neq 0, \hat{\nabla}^{n} R \neq 0
$$

where $\hat{\nabla}$ denotes the covariant derivative of $(1,0)$-type induced from $\nabla$ (see Section 3 for the defintion). Moreover, Takagi classified Kähler $C$-spaces with $n=2$ (Hermitian symmetric spaces of compact type are characterized as Kähler $C$-spaces with $n=1$ ).

However, there is a mistake in deduction to lead a certain formula. The purpose of this paper is to correct the mistake and to classify Kähler $C$-spaces with $n=2$. Moreover, in Section 5 , we shall classify Kähler $C$-spaces with $n=3$.

The author would like to thank Prof. R. Takagi for his kind advice and continuous encouragement.

\section{Preliminaries}

Let $G$ be a Lie group and $K$ a closed subgroup of $G$. Let $g$ and $\mathfrak{k}$ be the Lie algebras of $G$ and $K$, respectively. Suppose that $\operatorname{Ad}(K)$ is compact. Then there exist an $\operatorname{Ad}(K)$-invariant decomposition $\mathfrak{g}=\mathfrak{f}+\mathfrak{p}$ of $\mathfrak{g}$ and an $\operatorname{Ad}(K)$-invariant scalar product $\langle$,$\rangle on \mathfrak{p}$. Then

$$
\begin{aligned}
{[\mathfrak{t}, \mathfrak{p}] } & \subset \mathfrak{p} \\
\langle[u, x], y\rangle+\langle[u, y], x\rangle & =0(u \in \mathfrak{k}, x, y \in \mathfrak{p}) .
\end{aligned}
$$

Moreover, under the canonical identification of $\mathfrak{p}$ with the tangent space $T_{o}(G / K)$ ( $o=\{K\}$ ) of homogeneous space $G / K$, the scalar product $\langle$,$\rangle can be extended to$ Received January 10. 1995. 
a $G$-invariant metric on $G / K$.

Let $\Lambda$ be the connection function of $(G / K,\langle\rangle$,$) (cf.[5]). Then for x, y \in \mathfrak{p}$,

$$
\Lambda(x)(y)=\frac{1}{2}[x, y]_{\mathfrak{p}}+U(x, y)
$$

where

$$
\langle U(x, y), z\rangle=\frac{1}{2}\left\{\left\langle[z, x]_{\mathfrak{p}}, y\right\rangle+\left\langle[z, y]_{\mathfrak{p}}, x\right\rangle\right\} \quad(z \in \mathfrak{p}) .
$$

Furthermore the curvature tensor $R$ is given by

$$
\begin{aligned}
R(x, y) z= & {[\Lambda(x), \Lambda(y)] z-\left[[x, y]_{\mathfrak{f}}, z\right] } \\
& -\Lambda\left([x, y]_{\mathfrak{p}}\right) z .
\end{aligned}
$$

In the remaining part of this section we describe irreducible Kähler $C$-spaces and recall some properties with respect to the connection functions (see [3] for example).

Let $\mathfrak{g}$ be a simple Lie algebra over $\mathbf{C}$ with $\operatorname{rk}(\mathfrak{g})=l$, and $\mathfrak{h}$ a Cartan subalgebra of $\mathfrak{g}, \Delta$ denotes the set of non-zero roots of $\mathfrak{g}$ with respect to $\mathfrak{h}$. For some lexicographic order we denote by $I I=\left\{\alpha_{1}, \ldots, \alpha_{l}\right\}$ the fundamental root system of $\Delta$. Moreover let $\Delta^{+}$be the set of positive roots of $\Delta$ with respect to the order. Since $\mathfrak{g}$ is simple, we can define $H_{\alpha} \in \mathfrak{h}(\alpha \in \Delta)$ by

$$
B\left(H, H_{\alpha}\right)=\alpha(H)(H \in \mathfrak{h})
$$

where $B$ is the Killing form of $g$. We choose root vectors $\left\{E_{\alpha}\right\}$ ( $\alpha \in \Delta$ ) so that for $\alpha, \beta \in \Delta$

$$
\begin{aligned}
& B\left(E_{\alpha}, E_{-\alpha}\right)=1, \\
& {\left[E_{\alpha}, E_{\beta}\right]=N_{\alpha, \beta} E_{\alpha+\beta}, N_{\alpha, \beta}=-N_{-\alpha,-\beta} \in \mathbf{R} .}
\end{aligned}
$$

Then $\left[E_{\alpha}, E_{-\alpha}\right]=H_{\alpha}$. Moreover the following hold (cf. [2]).

$$
\begin{gathered}
N_{\alpha, \beta}=N_{\beta, \gamma}=N_{\gamma, \alpha} \text { if } \alpha+\beta+\gamma=0 \\
N_{\alpha, \beta} N_{\gamma, \delta}+N_{\beta, \gamma} N_{\alpha, \gamma}+N_{\gamma, \alpha} N_{\beta, \delta}=0,
\end{gathered}
$$

if $\alpha+\beta+\gamma+\delta=0$ (no two of which have sum 0 ). Let $\{\beta+n \alpha ; p \leq n \leq q\}$ be the $\alpha$-series containing $\beta$. Then

$$
\left(N_{\alpha, \beta}\right)^{2}=\frac{q(1-p)}{2} \alpha\left(H_{\alpha}\right), \frac{2 \alpha\left(H_{\beta}\right)}{\alpha\left(H_{\alpha}\right)}=-(p+q) .
$$


As is well-known, the subalgebra $\mathfrak{g}_{u}$ of $\mathfrak{g}$ defined in the following is a compact real form of $g$ :

$$
\mathfrak{g}_{u}=\sum_{\alpha \in \Delta^{+}} \mathbf{R} \sqrt{-1} H_{\alpha}+\sum_{\alpha \in \Delta^{+}}\left(\mathbf{R} A_{\alpha}+\mathbf{R} B_{\alpha}\right),
$$

where $A_{\alpha}=E_{\alpha}-E_{-\alpha}$ and $B_{\alpha}=\sqrt{-1}\left(E_{\alpha}+E_{-\alpha}\right)$.

Consider a non-empty subset $\Psi=\left\{\alpha_{i_{1}}, \ldots, \alpha_{i_{r}}\right\}$ of $I I$. Set

$$
\Delta^{+}(\Psi)=\left\{\alpha=\sum_{j=1}^{l} n_{j} \alpha_{j} \in \Delta^{+} ; n_{i_{k}}>0 \text { for some } \alpha_{i_{k}} \in \Psi\right\} \text {. }
$$

Then we define a subalgebra $\mathfrak{L}_{w}$ as follows:

$$
\mathfrak{k}_{\Psi}=\sum_{\alpha \in \Delta^{+}} \mathbf{R} \sqrt{-1} H_{\alpha}+\sum_{\alpha \in \Delta^{+}-\Delta^{+}(\Psi)}\left(\mathbf{R} A_{\alpha}+\mathbf{R} B_{\alpha}\right) .
$$

Let $G_{u}$ and $K_{\Psi}$ be a simply connected Lie group and its connected closed subgroup which correspond to $g_{u}$ and $\mathfrak{l}_{\Psi}$ respectively. Then $G_{u} / K_{\Psi}$ is an irreducible $C$-space.

Put

$$
\mathfrak{p}=\sum_{\alpha \in \Delta^{+}(\Psi)}\left(\mathbf{R} A_{\alpha}+\mathbf{R} B_{\alpha}\right) .
$$

Then $\mathfrak{g}_{u}=\mathfrak{l}_{\Psi}+\mathfrak{p}$ (direct sum) and the tangent space $T_{o}\left(G_{u} / K_{\Psi}\right)$ of $G_{u} / K_{\Psi}$ at $o=\left\{K_{\Psi}\right\}$ is identified with $\mathfrak{p}$. Then a complex structure $I$ is given at $o$ by

$$
I\left(A_{\alpha}\right)=B_{\alpha}, I\left(B_{\alpha}\right)=-A_{\alpha}\left(\alpha \in \Delta^{+}(\Psi)\right) .
$$

We set

$$
\mathfrak{p}^{ \pm}=\sum_{\alpha \in \Delta^{+}(\Psi)} \mathbf{C} E_{ \pm \alpha} .
$$

Then we have $\mathfrak{p}^{ \pm}=\left\{X \in \mathfrak{p}^{\mathbf{C}} ; I(X)= \pm \sqrt{-} \overline{1} X\right\}$. An element of $\mathfrak{p}^{+}$is said to be of $(1,0)$-type.

Define a mapping $p: \Delta^{+}(\Psi) \rightarrow \mathbf{Z}^{r}$ as follows:

$$
p(\alpha)=\left(n_{i_{1}}(\alpha), \ldots, n_{i_{r}}(\alpha)\right) \text { for } \alpha=\sum_{i=1}^{l} n_{i}(\alpha) \alpha_{i} \in \Delta^{+}(\Psi) .
$$

Let $\omega^{\alpha}$ and $\bar{\omega}^{\alpha}$ be the dual forms of $E_{\alpha}$ and $E_{-\alpha}$, respectively. Then any $G_{u}$-invariant Kähler metric $g$ is given at $o$ by

$$
g=-2 \sum_{\alpha \in \Delta^{+}(\varphi)}(c \cdot p(\alpha)) \omega^{\alpha} \cdot \bar{\omega}^{\alpha}
$$


where $c=\left(c_{1}, \ldots, c_{r}\right)\left(c_{j}>0\right)$ and $c \cdot p(\alpha)=\sum_{j=1}^{r} c_{j} n_{i_{j}}(\alpha)$. Conversely, any bilinear form $-2 \sum_{\alpha}(c \cdot p(\alpha)) \omega^{\alpha} \cdot \bar{\omega}^{\alpha}$ on $\mathfrak{p}^{\mathbf{C}} \times \mathfrak{p}^{\mathbf{C}}$ can be extended to a $G_{u}$-invariant metric on $G_{u} / K_{\psi}$.

In the following we regard the metrics, connections and tensors as ones extended naturally over $\mathbf{C}$.

In [3] the connection functions of Kähler spaces are determined.

For $\alpha, \beta \in \Delta$ we write $p(\alpha)>p(\beta)$ if $n_{i_{k}}(\alpha) \geq n_{i_{k}}(\beta)(k=1, \ldots, r)$ and $n_{i_{j}}(\alpha)>n_{i,}(\beta)$ for some $j$. Then

Lemma 1.1. For $\alpha \in \Delta^{+}(\Psi)$, identify $\alpha$ with $E_{\alpha}$ and $\bar{\alpha}$ with $E_{-\alpha}$. Then

$$
\begin{aligned}
\Lambda(\alpha)(\beta) & =\frac{c \cdot p(\beta)}{c \cdot p(\alpha+\beta)}[\alpha, \beta] \\
\Lambda(\bar{\alpha})(\beta) & = \begin{cases}{[\bar{\alpha}, \beta]} & p(\alpha)<p(\beta) \\
0 & \text { otherwise }\end{cases} \\
\Lambda(\alpha)(\bar{\beta}) & = \begin{cases}{[\alpha, \bar{\beta}]} & p(\alpha)<p(\beta) \\
0 & \text { otherwise }\end{cases} \\
\Lambda(\bar{\alpha})(\bar{\beta}) & =\frac{c \cdot p(\beta)}{c \cdot p(\alpha+\beta)}[\bar{\alpha}, \bar{\beta}] .
\end{aligned}
$$

\section{Covariant derivatives on homogeneous spaces}

In this section we shall write the Levi-Civita connections of Riemannian homogeneous spaces in terms of the Lie algebras.

Let $(M, g)$ be an $n$-dimensional Riemannian manifold and $\nabla$ the Levi-Civita connection of $(M, g)$. Let $\left\{e_{1}, \ldots, e_{n}\right\}$ be local orthonormal frame fields and $\left\{\omega^{1}, \ldots, \omega^{n}\right\}$ their dual 1 -forms. Associated with $\left\{e_{1}, \ldots, e_{n}\right\}$, there uniquely exist local 1 -forms $\left\{\omega_{i}^{j}\right\}(i, j=1, \ldots, n)$, which are called the connection forms, such that

$$
\begin{gathered}
\omega_{i}^{\jmath}+\omega_{j}^{i}=0 \\
d \omega^{i}+\sum_{j=1}^{n} \omega_{j}^{\imath} \wedge \omega^{j}=0 .
\end{gathered}
$$

Then the following holds.

$$
\nabla_{e_{i}} e_{j}=\sum_{k=1}^{n} \omega_{j}^{k}\left(e_{\imath}\right) e_{k}
$$


(see [4]).

Next, let $(G / K,\langle\rangle$,$) be a homogeneous space with a G$-invariant metric $\langle$,$\rangle as stated in Section 1$.

Let $\pi: G \rightarrow G / K$ be the canonical projection and $W$ an open subset in $\mathfrak{p}$ such that $0 \in W$ and the mapping

$$
\pi \circ \exp : W \rightarrow \pi(\exp W)
$$

is diffeomorphic. Let $\left\{e_{\alpha}\right\}_{\alpha \in A}$ be a basis of ${ }^{\prime}$ and $\left\{e_{i}\right\}_{i \in I}$ an orthonormal basis of $(\mathfrak{p},\langle\rangle$,$) . In this section we use the following convention on the range of indices,$ unless otherwise stated:

$$
\begin{aligned}
& i, j, k, \ldots \in I, \alpha, \beta, \gamma, \ldots \in A, \\
& p, q, r, \ldots \in I \cup A .
\end{aligned}
$$

Let $\left\{X_{\alpha}\right\}$ and $\left\{X_{i}\right\}$ be the left invariant vector fields on $G$ such that $\left(X_{\alpha}\right)_{e}=e_{\alpha}$ and $\left(X_{i}\right)_{e}=e_{i}(e$ is the identity of $G$ ). Furthermore we define an orthonormal frame field $\left\{E_{i}\right\}$ on $\pi(\exp W)$ and the mapping $\mu: \pi(\exp W) \rightarrow \exp W$ as follows:

$$
\begin{aligned}
& \left(E_{i}\right)_{\pi(\exp x)}=\tau(\exp x)_{*}\left(e_{\imath}\right) \\
& \mu(\pi(\exp x))=\exp x \quad(x \in W),
\end{aligned}
$$

where $\tau(g)(g \in G)$ denotes the left transformation of $G / K$. Then since $\pi_{*}\left(X_{i}\right)=E_{\imath}, \pi_{*}\left(X_{\alpha}\right)=0$ and $\pi_{*} \mu_{*}=$ id, we can put

$$
\mu_{*}\left(E_{i}\right)=X_{i}+\sum_{\alpha} \eta_{\alpha i} X_{\alpha}
$$

Let $\left\{\omega^{\alpha}\right\},\left\{\omega^{i}\right\}$ and $\left\{\theta^{i}\right\}$ be the dual 1 -forms of $\left\{X_{\alpha}\right\},\left\{X_{i}\right\}$ and $\left\{E_{\imath}\right\}$, respectively. Then it is easy to see

$$
\mu^{*}\left(\omega^{i}\right)=\theta^{i}
$$

Set $\left[X_{p}, X_{q}\right]=\sum_{r} c_{p q}^{r} X_{r}$. Then the following is known as the equation of Maurer-Cartan (cf. [4]).

$$
d \omega^{p}=-\frac{1}{2} \sum_{q, r} c_{q r}^{p} \omega^{q} \wedge \omega^{r}
$$

For the sake of completeness we show the following well-known fact.

Lemma 2.1 Let $\left\{\theta_{j}{ }^{i}\right\}$ be the connection forms of $(G / K,\langle\rangle$,$) associated with$ $\left\{E_{i}\right\}$. Then 


$$
\theta_{j}{ }^{\imath}=-\mu^{*}\left\{\sum_{\alpha} c_{j \alpha}{ }^{i} \omega^{\alpha}+\frac{1}{2} \sum_{k}\left(c_{j k}{ }^{2}-c_{i k}{ }^{j}-c_{i j}{ }^{k}\right) \omega^{k}\right\}
$$

Proof. It follows from (1.1) and (1.2) that

$$
c_{j \alpha}{ }^{\beta}=0, \quad c_{i \alpha}{ }^{\prime}+c_{i \alpha}{ }^{\jmath}=0 .
$$

Moreover since $\operatorname{lo}$ is subalgebra of $\mathfrak{g}$, we get

$$
c_{\alpha \beta}{ }^{\imath}=0 .
$$

From equations (2.5), (2.6), (2.7) and (2.8) it follows that

$$
\begin{aligned}
d \theta^{i} & =\mu^{*} d \omega^{i} \\
& =-\sum_{j} \mu^{*}\left\{\sum_{\alpha} c_{j \alpha}{ }^{i} \omega^{j} \wedge \omega^{\alpha}+\frac{1}{2} \sum_{k}\left(c_{j k}{ }^{2}-c_{i k}{ }^{j}-c_{i j}{ }^{k}\right) \omega^{j} \wedge \omega^{k}\right\} \\
& =\sum_{j} \mu^{*}\left\{\sum_{\alpha} c_{j \alpha}{ }^{i} \omega^{\alpha}+\frac{1}{2} \sum_{k}\left(c_{j k}{ }^{2}-c_{i k}{ }^{j}-c_{i j}{ }^{k}\right) \omega^{k}\right\} \wedge \theta^{j}
\end{aligned}
$$

(note that $\sum_{j, k}\left(c_{i j}{ }^{k}+c_{i k}{ }^{j}\right) \omega^{j} \wedge \omega^{k}=0$ ).

Put $\theta_{j}{ }^{i}=-\mu^{*}\left\{\sum_{\alpha} c_{j \alpha}{ }^{i} \omega^{\alpha}+(1 / 2) \sum_{k}\left(c_{j k}{ }^{i}-c_{i k}{ }^{j}-c_{i j}{ }^{k}\right) \omega^{k}\right\}$. Then it is easy to see $\theta_{j}^{i}+\theta_{i}^{j}=0$.

Consequently, by (2.1) and (2.2), the connection forms coincide with $\left\{\theta_{j}{ }^{i}\right\}$.

By (2.3), (2.4) and the above lemma, we have the following.

PROPOSITION 2.2.

$$
\nabla_{E_{i}} E_{j}=\sum_{k}\left\{\sum_{\alpha} c_{\alpha j}{ }^{k} \eta_{\alpha i}+\frac{1}{2}\left(c_{\imath j}{ }^{k}-c_{\imath k}{ }^{j}-c_{j k}{ }^{i}\right)\right\} E_{k} .
$$

Next we shall rewrite Proposition 2.2 in terms of the bracket operation [, ] of $\mathfrak{g}$.

For $x \in W$, we define $z_{x}{ }^{i}(t) \in W$ and $h_{x}{ }^{i}(t) \in K(t \in \mathbf{R},|t|$ : small enough) by the following:

$$
\exp x \cdot \exp t e_{\imath}=\exp z_{x}{ }^{2}(t) \cdot h_{x}{ }^{i}(t)
$$

with $z_{x}^{i}(0)=x$ and $h_{x}^{i}(0)=e$. Then

$$
\begin{aligned}
\mu_{*}\left(E_{i}\right)_{\pi(\exp x)} & =\left.\frac{d}{d t}\right|_{0} \mu\left(\pi\left(\exp x \cdot \exp t e_{i}\right)\right) \\
& =\left.\frac{d}{d t}\right|_{0} \mu\left(\pi\left(\exp z_{x}{ }^{\imath}(t)\right)\right)
\end{aligned}
$$




$$
=\left(\exp _{*}\right)_{x}\left(\left.\frac{d}{d t}\right|_{0} z_{x}^{i}(t)\right)
$$

Here, the differential map $\exp _{*}$ of exp has the following form (see [2]).

Lemma 2.3. Let $x, y \in$ g. Then

$$
\left(\exp _{*}\right)_{x}(y)=\left(L_{\exp x}\right)_{*} \circ \Phi_{x}(y),
$$

where $\Phi_{x}(y)=\sum_{n=0}^{\infty} \frac{(-1)^{n}}{(n+1) !}(\operatorname{ad} x)^{n}(y)$.

Thus we have

$$
\mu_{*}\left(E_{i}\right)_{\pi(\exp x)}=\left(L_{\exp x}\right)_{*} \Phi_{x}\left(\left.\frac{d}{d t}\right|_{0} z_{x}^{i}(t)\right) .
$$

On the other hand, (2.9) and Lemma 2.3 give

$$
\begin{aligned}
\left(L_{\exp x}\right)_{*} \circ \Phi_{x}\left(\left.\frac{d}{d t}\right|_{0} z_{x}{ }^{i}(t)\right)= & \left(L_{\exp x}\right)_{*}\left(e_{\imath}\right) \\
& -\left(L_{\exp x}\right)_{*}\left(\left.\frac{d}{d t}\right|_{0} h_{x}{ }^{i}(t)\right) .
\end{aligned}
$$

Considering (2.4), (2.10) and (2.11), we obtain

$$
\left.\frac{d}{d t}\right|_{0} h_{x}^{i}(t)=-\sum_{\alpha} \eta_{\alpha i}(\exp x) e_{\alpha} .
$$

Therefore, by (2.12) and Proposition 2.2, we have

$$
\left(\nabla_{E_{t}} E_{j}\right)_{\pi(\exp x)}=\tau(\exp x)_{*}\left\{\Lambda\left(e_{i}\right)\left(e_{j}\right)-\left[\left.\frac{d}{d t}\right|_{0} h_{x}^{i}(t), e_{j}\right]\right\}
$$

Remark. For $x \in \mathfrak{p}(|x|:$ small), the mapping

$$
p_{\mathfrak{p}} \circ \Phi_{x}: \mathfrak{p} \rightarrow \mathfrak{p}
$$

is an isomorphism $\left(p_{\mathfrak{p}}: \mathfrak{g} \rightarrow \mathfrak{p}\right.$ denotes the canonical projection.). So we can assume that for each $x \in W$ the mapping $p_{\mathfrak{p}} \circ \Phi_{x}$ is an isomorphism. Therefore we can regard the equation $(2.11)$ as a characterization of $\left.\frac{d}{d t}\right|_{0} z_{x}{ }^{2}(t)(\in \mathfrak{p})$ and $\left.\frac{d}{d t}\right|_{0} h_{x}^{i}(t)(\in \mathfrak{k})$.

For $X \in \mathfrak{p}$, we denote by $X_{*}$ the vector field on $\pi(\exp W)$ defined by 


$$
\left(X_{*}\right)_{\pi(\exp x)}=\tau(\exp x)_{*}(X) .
$$

Then the following theorem is easily derived from the above arguments.

Theorem 2.4. Let $x \in W$ and $X, Y \in \mathfrak{p}$, Then

$$
\left(\nabla_{X *} Y_{*}\right)_{\pi(\exp x)}=\tau(\exp x)_{*}\left\{\Lambda(X)(Y)-\left[h_{x}(X), Y\right]\right\} .
$$

Here $h_{x}(X)=-p_{\mathfrak{k}} \circ \Phi_{x} \circ\left(p_{\mathfrak{p}} \circ \Phi_{x}\right)^{-1}(X)\left(p_{\mathfrak{k}}: \mathfrak{g} \rightarrow \mathfrak{f}\right.$ denotes the canonical projection).

\section{Covariant derivatives on Kähler $C$-spaces}

In this section we shall write higher covariant derivatives of $(1,0)$-type on Kähler $C$-spaces in terms of the connection functions.

Let $\left(G_{u} / K_{\Psi},\langle\rangle,\right)$ be a Kählerian $C$-space as stated in Section 1 . For $\alpha \in$ $\Delta^{+}(\Psi)$, since $\alpha=(1 / 2)\left(A_{\alpha}-\sqrt{-1} B_{\alpha}\right)$ (under the identification $E_{\alpha}$ with $a$ ), we have

$$
\alpha_{*}=\frac{1}{2}\left(A_{\alpha *}-\sqrt{-1} B_{\alpha *}\right)
$$

At first we calculate the value of $\nabla^{n}\left(X_{*} ; \alpha_{1}{ }^{*}, \ldots, \alpha_{n}{ }^{*}\right)$ at $o\left(X \in \mathfrak{p}^{\mathbf{C}}, \alpha_{\imath} \in\right.$ $\left.\Delta^{+}(\Psi)\right)$.

Let $X_{i}(i=1, \ldots, n)$ be one of $\left\{A_{i}, B_{i}\right\}\left(A_{i}=A_{\alpha_{i}}, B_{i}=B_{\alpha_{i}}\right)$. For $s_{1}, \ldots, s_{n}$ $\in \mathbf{R}\left(\left|s_{\imath}\right|\right.$ : small enough), we define $z^{i}\left(s_{1}, \ldots, s_{i}\right) \in W(1 \leq i \leq n)$ inductively as follows:

$$
\begin{aligned}
& z^{1}\left(s_{1}\right)=s_{1} X_{1} \\
& \pi\left(\exp z^{i}\left(s_{1}, \ldots, s_{i}\right)\right)=\pi\left(\exp z^{i-1}\left(s_{1}, \ldots, s_{i-1}\right) \exp s_{i} X_{i}\right)
\end{aligned}
$$

Then

$$
z^{2}\left(s_{1}, \ldots, s_{\imath-1}, 0\right)=z^{i-1}\left(s_{1}, \ldots, s_{i-1}\right) .
$$

Then it follows Lemma 2.3, (3.1) and (3.2) that

$$
X_{i}=p_{\mathfrak{p}} \circ \Phi_{z^{i-1}\left(s_{1}, \ldots, s_{i-1}\right)}\left(\left.\frac{\partial}{\partial s_{i}}\right|_{0} z^{i}\left(s_{1}, \ldots, s_{i}\right)\right)
$$

From Theorem 2.4 we have

$$
\begin{aligned}
& \left(\nabla_{X_{n *}} X_{*}\right)_{\pi\left(\exp z^{n}\left(s_{1}, \ldots, s_{n-1}, 0\right)\right)} \\
& =\tau\left(\exp z^{n-1}\left(s_{1}, \ldots, s_{n-1}\right)\right)_{*}\left\{\Lambda\left(X_{n}\right)(X)-\left[h_{n-1}\left(s_{1}, \ldots, s_{n-1}\right), X\right]\right\}
\end{aligned}
$$


where

$$
\begin{aligned}
& h_{n-1}\left(s_{1}, \ldots, s_{n-1}\right)=-p_{\mathfrak{£}} \circ \Phi_{z^{n-1}\left(s_{1}, \ldots, s_{n-1}\right)}\left(V_{n-1}\left(s_{1}, \ldots, s_{n-1}\right)\right) \\
& X_{n}=p_{\mathfrak{p}} \circ \Phi_{z^{n-1}\left(s_{1}, \ldots, s_{n-1}\right)}\left(V_{n-1}\left(s_{1}, \ldots, s_{n-1}\right)\right) .
\end{aligned}
$$

Thus, by (3.3) we get

$$
V_{n-1}=\left.\frac{\partial}{\partial s_{n}}\right|_{0} z^{n}
$$

Similarly, we have by (3.4) and Theorem 2.4

$$
\begin{aligned}
( & \left.\nabla_{X_{n-1 *}} \nabla_{X_{n *}} X_{*}\right)_{\pi\left(\exp z^{n-2}\left(s_{1}, \ldots, s_{n-2}\right)\right)} \\
= & \tau\left(\exp z^{n-2}\left(s_{1}, \ldots, s_{n-2}\right)\right)_{*}\left\{\Lambda\left(X_{n-1}\right) \Lambda\left(X_{n}\right)(X)\right. \\
& -\Lambda\left(X_{n-1}\right)\left(\left[h_{n-1}\left(s_{1}, \ldots, s_{n-2}, 0\right), X\right]-\left.\frac{\partial}{\partial s_{n-1}}\right|_{0}\left[h_{n-1}\left(s_{1}, \ldots, s_{n-1}\right), X\right]\right) \\
& \left.-\left[h_{n-2}\left(s_{1}, \ldots, s_{n-2}\right), \Lambda\left(X_{n}\right)(X)-\left[h_{n-1}\left(s_{1}, \ldots, s_{n-2}, 0\right), X\right]\right]\right\}
\end{aligned}
$$

where

$$
\begin{aligned}
& h_{n-2}\left(s_{1}, \ldots, s_{n-2}\right)=-p_{\mathfrak{k}} \circ \Phi_{z^{n-2}\left(s_{1}, \ldots, s_{n-2}\right)}\left(\left.\frac{\partial}{\partial s_{n-1}}\right|_{0} z^{n-1}\right) \\
& X_{n-1}=p_{\mathfrak{k}} \circ \Phi_{z^{n-2}\left(s_{1}, \ldots, s_{n-2}\right)}\left(\left.\frac{\partial}{\partial s_{n-1}}\right|_{0} z^{n-1}\right) .
\end{aligned}
$$

Therefore, by induction, we can see

$$
\begin{aligned}
& \left(\nabla_{X_{1 *}} \cdots \nabla_{X_{n *}} X_{*}\right)_{o} \\
& =\Lambda\left(X_{1}\right) \cdots \Lambda\left(X_{n}\right)(X) \\
& \quad+\left\{\text { terms containing }\left.\frac{\partial^{r}}{\partial s_{i_{1}} \cdots \partial s_{i_{r}}}\right|_{s_{1}=\cdots=s_{k-1}=0} h_{k-1}\left(s_{1}, \ldots, s_{k-1}\right)\right. \\
& \quad \text { for some } k, r\} .
\end{aligned}
$$

Here

$$
\begin{gathered}
h_{k-1}\left(s_{1}, \ldots, s_{k-1}\right)=-p_{\mathfrak{E}} \circ \Phi_{z^{k-1}\left(s_{1}, \ldots, s_{k-1}\right)}\left(\left.\frac{\partial}{\partial s_{k}}\right|_{0} z^{k}\right) \\
X_{k}=p_{\mathfrak{p}} \circ \Phi_{z^{k-1}\left(s_{1}, ., s_{k-1}\right)}\left(\left.\frac{\partial}{\partial s_{k}}\right|_{0} z^{k}\right) .
\end{gathered}
$$


Lemma 3.1. Expand $z^{n}\left(s_{1}, \ldots, s_{n}\right)$ as

$$
z^{n}\left(s_{1}, \ldots, s_{n}\right)=\sum_{i_{1}, \cdots, i_{k}} s_{i_{1}} \cdots s_{i_{k}} a_{i_{1}, \cdots, i_{k}} .
$$

Then there exists a multi-linear function

$$
F_{i_{1}, \cdots, i_{k}}:\left(\mathfrak{p}^{\mathbf{C}}\right)^{k} \rightarrow \mathfrak{p}^{\mathbf{C}}
$$

such that

$$
a_{i_{1}, \cdots, i_{k}}=F_{i_{1}, \cdots, i_{k}}\left(X_{i_{1}}, \ldots, X_{i_{k}}\right)
$$

Proof. At first we note that $z^{n}(0, \ldots, 0)=0$ and

$$
\begin{aligned}
& z^{n}\left(s_{1}, \ldots, s_{i}, 0, \ldots, 0\right)=z^{i}\left(s_{1}, \ldots, s_{i}\right), \\
& z^{n}\left(0, \ldots, 0, s_{i}, 0, \ldots, 0\right)=s_{i} X_{i} .
\end{aligned}
$$

We prove the lemma by induction.

Assume that for any $r$-tuple $\left(i_{1}, \ldots, i_{r}\right)\left(1 \leq r \leq k, i_{1}<\cdots<i_{r}\right)$ there exists $r$-linear function $F_{i_{1}, \cdots, i_{r}}$, such that

$$
a_{i_{1}, \cdots, i_{r}}=F_{i_{1}, \cdots, i_{r}}\left(X_{i_{1}}, \ldots, X_{i_{r}}\right) .
$$

Then for any $(k+1)$-tuple $\left(j_{1}, \ldots, j_{k}, j_{k+1}\right)\left(j_{1}<\cdots<j_{k+1}\right)$ it follows from (3.9) that

$$
X_{j_{k+1}}=p_{\mathfrak{p}} \circ \Phi_{z^{j_{k+1}\left(s_{1}, \ldots, s_{j_{k+1}-1}, 0\right)}}\left(\left.\frac{\partial}{\partial s_{j_{k+1}}}\right|_{0} z^{j_{k+1}}\right) .
$$

Considering the $\left(s_{j_{1}} \cdots s_{j_{k}}\right)$-term of the above equation, we have

$$
0=a_{j_{1}, \cdots, j_{k+1}}+\sum_{l=1}^{k} \frac{(-1)^{l}}{(l+1) !} \sum_{J_{1}, \cdots, J_{l+1}}\left[a_{J_{1}},\left[\cdots\left[a_{J_{l}}, a_{J_{l+1}}\right] \cdots\right]_{\mathfrak{p}} .\right.
$$

Here, each $J_{p}, 1 \leq p \leq l+1$, is a subset of $\left\{j_{1}, \ldots, j_{k+1}\right\}$ such that $J_{p} \cap J_{q}=\emptyset$ $(p \neq q), J_{p} \subset\left\{j_{1}, \ldots, j_{k}\right\}$ for $1 \leq p \leq l$ and

$$
J_{1} \cup \cdots \cup J_{l} \cup J_{l+1}=\left\{j_{1}, \ldots, j_{k+1}\right\}
$$

Therefore, by the inductive assumption, the $\left(s_{j_{1}} \cdots s_{j_{k+1}}\right)$-term of $z^{n}$ is written as in the lemma. This completes the proof of the lemma.

Let $h^{r}{ }_{j_{1}, \ldots, j_{k}}$ be the $\left(s_{j_{1}} \cdots s_{j_{k}}\right)$-term of $h_{r}\left(s_{1}, \ldots, s_{r}\right)$. Then, by $(3.8)$ and the proof of Lemma 3.1, we have. 


$$
\begin{aligned}
& h_{j_{1}, \ldots, j_{k}}^{r} \\
& =-\sum_{l=1}^{k} \sum_{J_{1}, \ldots, J_{l+1}} \frac{(-1)^{l}}{(l+1) !}\left[a_{J_{1}},\left[\cdots,\left[a_{J_{l}}, a_{J_{l+1}}\right] \cdots\right]_{\mathfrak{f}} .\right.
\end{aligned}
$$

Thus, by Lemma 3.1 and (3.10), there exists $k$-linear map

$$
H^{r}{ }_{j_{1}, \ldots, j_{k}}:\left(\mathfrak{p}^{\mathbf{C}}\right)^{k} \rightarrow \mathfrak{f}^{\mathbf{C}}
$$

such that

$$
h_{j_{1}, \ldots, j_{k}}^{r}=H_{j_{1}, \ldots, J_{k}}^{r}\left(X_{j_{1}}, \ldots, X_{j_{k}}\right) .
$$

Therefore (3.7) gives

$$
\begin{aligned}
& \left(\nabla_{\alpha_{1 *}} \cdots \nabla_{\alpha_{n *}} X_{*}\right)_{o} \\
& =\Lambda\left(\alpha_{1}\right) \cdots \Lambda\left(\alpha_{n}\right)(X) \\
& \quad+\left\{\text { terms containing } H_{j_{1}, \ldots, j_{k}}^{r}\left(\alpha_{j_{1}}, \ldots, \alpha_{j_{k}}\right)\right\} .
\end{aligned}
$$

For $\alpha, \beta \in \Delta^{+}(\Psi)$, it is obvious that $\alpha+\beta \in \Delta^{+}(\Psi)$ if $\alpha+\beta \in \Delta$. Considering the form of $H^{r}{ }_{j_{1}, \ldots, j_{k}}$, it is easy to see that

$$
H^{r}{ }_{j_{1}, \ldots, j_{k}}\left(\alpha_{j_{1}}, \ldots, \alpha_{j_{k}}\right) \in \mathfrak{p}^{+} .
$$

We have thus the following.

Proposition 3.2. Let $\alpha_{\imath}(i=1, \ldots, n)$ be in $\Delta^{+}(\Psi)$ and $X \in \mathfrak{p}^{\mathbf{C}}$. Then

$$
\left(\nabla_{\alpha_{1 *}} \cdots \nabla_{\alpha_{n *}} X_{*}\right)_{o}=\Lambda\left(\alpha_{1}\right) \cdots \Lambda\left(\alpha_{n}\right)(X) .
$$

Remark 3.3. By similar argument as in the above, we can prove that

$$
\left(\nabla_{\nabla_{\alpha *} \beta_{*}} \cdots\right)_{o}=\Lambda\left(\Lambda\left(\alpha_{1}\right)(\beta)\right)(\cdots)
$$

for $\alpha, \beta, \cdots \in \Delta^{+}(\Psi)$.

Now, we define $\Lambda^{n} R$ inductively as follows.

$(\Lambda R)(X, Y, Z ; T)$

$$
\begin{aligned}
= & \Lambda(T)(R(X, Y) Z)-R(\Lambda(T)(X), Y) Z-R(X, \Lambda(T)(X)) Z \\
& -R(X, Y) \Lambda(T)(Z), \\
& \left(\Lambda^{n} R\right)\left(X, Y, Z ; T_{1}, \ldots, T_{n}\right) \\
= & \Lambda\left(T_{n}\right)\left(\left(\Lambda^{n-1} R\right)\left(X, Y, Z ; T_{1}, \ldots, T_{n-1}\right)\right) \\
& -\left(\Lambda^{n-1} R\right)\left(\Lambda\left(T_{n}\right)(X), Y, Z ; T_{1}, \ldots, T_{n-1}\right)-\left(\Lambda^{n-1} R\right)\left(X, \Lambda\left(T_{n}\right)(Y),\right. \\
& \left.Z ; T_{1}, \ldots, T_{n-1}\right)-\left(\Lambda^{n-1} R\right)\left(X, Y, \Lambda\left(T_{n}\right)(Z) ; T_{1}, \ldots, T_{n-1}\right)
\end{aligned}
$$




$$
-\sum_{i=1}^{n-1}\left(\Lambda^{n-1} R\right)\left(X, Y, Z ; T_{1}, \ldots, \Lambda\left(T_{n}\right)\left(T_{i}\right), \ldots, T_{n-1}\right) .
$$

Here $X, \ldots, T_{n} \in \mathfrak{p}^{\mathbf{C}}$.

Since

$$
R\left(\alpha_{*}, \beta_{*}\right) \gamma_{*}=(R(\alpha, \beta) \gamma)_{*},
$$

Proposition 3.2 and Remark 3.3 give the following Theorem which is the correction of (2.11) and (3.11) of [6].

Theorem 3.4. Let $X, Y, Z \in \mathfrak{p}^{\mathbf{C}}$ and $\delta_{1}, \ldots, \delta_{n} \in \Delta^{+}(\Psi)$. Then

$$
\left(\nabla^{n} R\right)\left(X, Y, Z ; \delta_{1}, \ldots, \delta_{n}\right)=\left(\Lambda^{n} R\right)\left(X, Y, Z ; \delta_{1}, \ldots, \delta_{n}\right)
$$

Corollary 3.5. Let $\alpha, \beta$, and $\gamma$ be in $\Delta$ such that $E_{\alpha}, E_{\beta}$ and $E_{\gamma}$ are elements of $\mathfrak{p}^{\mathbf{C}}$. Moreover, let $\delta_{1}, \ldots, \delta_{n}$ be in $\Delta^{+}(\Psi)$. Then

$$
\left(\nabla^{n} R\right)\left(\alpha, \beta, \gamma ; \delta_{1}, \ldots, \delta_{n}\right) \in \mathbf{C} E_{\alpha+\beta+\gamma+\delta_{1}+\cdots+\delta_{n}} .
$$

We denote by $\hat{\nabla}$ the covariant derivative in the direction of $\mathfrak{p}^{+}$. Then, from Corollary 3.5, there is a number $n$ such that $\hat{\nabla}^{n} R=0$ and $\hat{\nabla}^{n-1} R \neq 0$. We call the integer $n$ the degree of $\left(G_{u} / K_{\Psi},\langle\rangle,\right)$. It is known that Hermitian symmetric spaces of compact type are characterized as Kähler $C$-spaces with degree one.

\section{Degree two}

In this section, using a similar method as in [6], we shall determine the class of Kählerian $C$-spaces with degree two.

Let $\alpha, \beta, \gamma, \delta$ and $\lambda$ be elements of $\Delta^{+}(\Psi)$. From Theorem 3.4, we have

$$
\begin{aligned}
& \left(\nabla^{2} R\right)(\alpha, \bar{\lambda}, \beta ; \gamma, \delta) \\
& =\Lambda(\delta) \Lambda(\gamma) R(\alpha, \bar{\lambda}) \beta-\Lambda(\Lambda(\delta) \gamma) R(\alpha, \bar{\lambda}) \beta-\Lambda(\gamma) R(\Lambda(\delta) \alpha, \bar{\lambda}) \beta \\
& -\Lambda(\gamma) R(\alpha, \Lambda(\delta) \bar{\lambda}) \beta-\Lambda(\gamma) R(\alpha, \bar{\lambda}) \Lambda(\delta) \beta-\Lambda(\delta) R(\Lambda(\gamma) \alpha, \bar{\lambda}) \beta \\
& +R(\Lambda(\Lambda(\delta) \gamma) \alpha, \bar{\lambda}) \beta+R(\Lambda(\gamma) \Lambda(\delta) \alpha, \bar{\lambda}) \beta+R(\Lambda(\gamma) \alpha, \Lambda(\delta) \bar{\lambda}) \beta \\
& +R(\Lambda(\gamma) \alpha, \bar{\lambda}) \Lambda(\delta) \beta-\Lambda(\delta) R(\alpha, \Lambda(\gamma) \bar{\lambda}) \beta+R(\Lambda(\delta) \alpha, \Lambda(\gamma) \bar{\lambda}) \beta \\
& +R(\alpha, \Lambda(\Lambda(\delta) \gamma) \bar{\lambda}) \beta+R(\alpha, \Lambda(\gamma) \Lambda(\delta) \bar{\lambda}) \beta+R(\alpha, \Lambda(\gamma) \bar{\lambda}) \Lambda(\delta) \beta \\
& -\Lambda(\delta) R(\alpha, \bar{\lambda}) \Lambda(\gamma) \beta+R(\Lambda(\delta) \alpha, \bar{\lambda}) \Lambda(\gamma) \beta+R(\alpha, \Lambda(\delta) \bar{\lambda}) \Lambda(\gamma) \beta \\
& +R(\alpha, \bar{\lambda}) \Lambda(\Lambda(\delta) \gamma) \beta+R(\alpha, \bar{\lambda}) \Lambda(\gamma) \Lambda(\delta) \beta .
\end{aligned}
$$


Lemma 4.1. Suppose that $\alpha, \beta\left(\in \Delta^{+}(\Psi)\right)(\alpha \neq \beta)$ satisfy the following conditions :

(1) $\alpha+\beta \in \Delta$, (2) $\alpha-\beta \notin \Delta$, (3) $2 \alpha+\beta \notin \Delta$, (4) $\alpha+2 \beta \notin \Delta$.

Then $\left(\nabla^{2} R\right)(\alpha, \overline{\alpha+\beta}, \beta ; \alpha, \beta) \neq 0$.

Proof. From (4.1) and the conditions in the lemma, we have

$\left(\nabla^{2} R\right)(\alpha, \overline{\alpha+\beta}, \beta ; \alpha, \beta)$

$$
\begin{aligned}
= & -\Lambda(\alpha) R(\Lambda(\beta) \alpha, \overline{\alpha+\beta}) \beta-\Lambda(\alpha) R(\alpha, \Lambda(\beta) \overline{\alpha+\beta}) \beta \\
& -\Lambda(\beta) R(\alpha, \Lambda(\alpha) \overline{\alpha+\beta}) \beta+R(\Lambda(\beta) \alpha, \Lambda(\alpha) \overline{a+\beta}) \beta-\Lambda(\beta) R(\alpha, \overline{\alpha+\beta}) \Lambda(\alpha) \beta \\
& +R(\Lambda(\beta) \alpha, \overline{\alpha+\beta}) \Lambda(\alpha) \beta+R(\alpha, \Lambda(\beta) \overline{a+\beta}) \Lambda(\alpha) \beta \\
= & \Lambda(\alpha)[[\Lambda(\beta) \alpha, \overline{\alpha+\beta}], \beta]+\Lambda(\alpha)\{\Lambda(\Lambda(\beta) \overline{\alpha+\beta}) \Lambda(\alpha) \beta+[[\alpha, \Lambda(\beta) \overline{\alpha+\beta}], \beta]\} \\
& +\Lambda(\beta) \Lambda(\Lambda(\alpha) \overline{\alpha+\beta}) \Lambda(\alpha) \beta-\Lambda([\Lambda(\beta) \alpha, \Lambda(\alpha) \overline{\alpha+\beta}]) \beta \\
& +\Lambda(\beta) \Lambda([\alpha, \overline{\alpha+\beta}]) \Lambda(\alpha) B-[[\Lambda(\beta) \alpha, \overline{\alpha+\beta}], \Lambda(\alpha) \beta] \\
& +\Lambda(\alpha) \Lambda(\Lambda(\beta) \overline{\alpha+\beta}) \Lambda(\alpha) \beta-[[\alpha, \Lambda(\beta) \overline{\alpha+\beta}], \Lambda(\alpha) \beta] .
\end{aligned}
$$

It follows from (1.6) and Lemma 1.1 that

$$
\begin{aligned}
& \left(\nabla^{2} R\right)(\alpha, \overline{\alpha+\beta}, \beta ; \alpha, \beta) \\
& =-\frac{(c \cdot p(\alpha))(c \cdot p(\beta))}{(c \cdot p(\alpha+\beta))^{2}}\left(N_{\alpha, \beta}\right)^{2} \beta\left(H_{\alpha+\beta}\right) \cdot(\alpha+\beta) \\
& \quad+2 \frac{(c \cdot p(\beta))^{2}}{(c \cdot p(\alpha+\beta))^{2}}\left(N_{\alpha, \beta}\right)^{2} N_{\beta,-(\alpha+\beta)} N_{-\alpha, \alpha+\beta} \cdot(\alpha+\beta) \\
& +\frac{c \cdot p(\beta)}{c \cdot p(\alpha+\beta)} N_{\alpha, \beta} N_{\beta,-(\alpha+\beta)} \beta\left(H_{\alpha}\right) \cdot(\alpha+\beta) \\
& -3 \frac{(c \cdot p(\alpha))(c \cdot p(\beta))}{(c \cdot p(\alpha+\beta))^{2}}\left(N_{\alpha, \beta}\right)^{2} N_{\alpha,-(\alpha+\beta)} N_{-\beta, \alpha+\beta} \cdot(\alpha+\beta) \\
& +\frac{(c \cdot p(\alpha))(c \cdot p(\beta))}{(c \cdot p(\alpha+\beta))^{2}}\left(N_{\alpha, \beta}\right)^{2}(\alpha+\beta)\left(H_{\alpha+\beta}\right) \cdot(\alpha+\beta) \\
& -\frac{c \cdot p(\beta)}{c \cdot p(\alpha+\beta)} N_{\alpha, \beta} N_{\beta,-(\alpha+\beta)} \alpha\left(H_{\alpha+\beta}\right) \cdot(\alpha+\beta) .
\end{aligned}
$$

It follows from (1.7) that

$$
N_{\beta,-(\alpha+\beta)}=-N_{\alpha,-(\alpha+\beta)}=N_{\alpha, \beta},
$$


form which we have

$$
\begin{aligned}
& \left(\nabla^{2} R\right)(\alpha, \overline{\alpha+\beta}, \beta ; \alpha, \beta) \\
& =\frac{c \cdot p(\beta)}{(c \cdot p(\alpha+\beta))}\left(N_{\alpha, \beta}\right)^{2}\left\{-\frac{c \cdot p(\alpha)}{(c \cdot p(\alpha+\beta))} \beta\left(H_{\alpha+\beta}\right)\right. \\
& \quad+2 \frac{(c \cdot p(\beta)}{(c \cdot p(\alpha+\beta))}\left(N_{\alpha, \beta}\right)^{2}+\beta\left(H_{\alpha}\right)-3 \frac{c \cdot p(\alpha)}{(c \cdot p(\alpha+\beta))}\left(N_{\alpha, \beta}\right)^{2} \\
& \left.\quad+\frac{c \cdot p(\alpha)}{(c \cdot p(\alpha+\beta))}(\alpha+\beta)\left(H_{\alpha+\beta}\right)-\alpha\left(H_{\alpha+\beta}\right)\right\} \cdot(\alpha+\beta) .
\end{aligned}
$$

From the conditions of Lemma 4.1, the $\alpha$-series containing $\beta$ is given by $\{\beta, \beta+\alpha\}$. Hence, by (1.9) we have

$$
\alpha\left(H_{\beta}\right)=-\frac{e}{2},\left(N_{\alpha, \beta}\right)^{2}=\frac{e}{2},
$$

where $e=\alpha\left(H_{\alpha}\right)=\beta\left(H_{\beta}\right)$. Therefore we have from (4.2)

$$
\left(\nabla^{2} R\right)(\alpha, \overline{\alpha+\beta}, \beta ; \alpha, \beta)=-\frac{e^{2}(c \cdot p(\alpha))(c \cdot p(\beta))}{(c \cdot p(\alpha+\beta))^{2}} \cdot(\alpha+\beta) .
$$

We have thus proved the lemma.

Now, we prove the following theorem.

Theorem 4.2. The only Kählerian $C$-spaces of which degrees are at most two are Hermitian symmetric spaces of compact type.

In the following we denote by $M(\mathfrak{g}, \Psi, g)$ the Kählerian $C$-space corresponding to $\Psi$. We show the theorem by case by case check.

The case where $\mathrm{g}$ is of type $A_{l}(l \geq 2)$.

We identify $\Delta$ with

$$
\left\{e_{i}-e_{j} ; 1 \leq i \neq j \leq l+1\right\}
$$

(for example, see [2]), where $\left\{e_{1}, \ldots, e_{l+1}\right\}$ is an orthonormal basis. Moreover, set $\alpha_{i}=e_{i}-e_{i+1}$. Then $M\left\{\mathrm{~g},\left\{\alpha_{i}\right\}, g\right)(i=1, \ldots, l)$ are Hermitian symmetric spaces.

Suppose that $\Psi$ contains $\alpha_{i}$ and $\alpha_{j}(i<j)$. Then $\alpha=\alpha_{1}+\cdots+\alpha_{i}$ and $\beta=$ $a_{i+1}+\cdots+\alpha_{l}$ are contained in $\Delta^{+}(\Psi)$. Furthermore, it is easy to see that $\alpha$ and 
$\beta$ satisfy the conditions (1), (2), (3) and (4) of Lemma 4.1. Thus the degree of $M(\mathfrak{g}, \Psi, g)$ is not equal to two.

The case where $\mathrm{g}$ is of type $B_{l}(l \geq 3)$.

$$
\Delta=\left\{ \pm e_{i}, \pm e_{i} \pm e_{j} ; 1 \leq i \neq j \leq l\right\}
$$

Set

$$
\alpha_{i}=e_{i}-e_{i+1}(1 \leq i \leq l-1), \alpha_{l}=e_{l} .
$$

In this case Hermitian symmetric spaces are $M\left(g,\left\{\alpha_{i}\right\}, g\right)(i=1, l)$.

Put

$$
\alpha=e_{1}-e_{l}=\alpha_{1}+\cdots+\alpha_{l-1}, \beta=e_{2}+e_{l-1}=\alpha_{2}+\cdots+\alpha_{l-1}+2 \alpha_{l} .
$$

Then we can easily see that $\alpha$ and $\beta$ satisfy the conditions of Lemma 4.1. Then Kählerian $C$-spaces of which degrees are at most two are only Hermitian symmetric spaces. In fact, if $\Psi$ contains some $\alpha_{i}(2 \leq i \leq l-1)$, then $\alpha, \beta \in \Delta^{+}(\Psi)$. Moreover, $\alpha, \beta \in \Delta^{+}\left(\left\{\alpha_{1}, \alpha_{l}\right\}\right)$.

The case where $\mathrm{g}$ is of type $C_{l}(l \geq 3)$.

$$
\Delta=\left\{ \pm 2 e_{i}, \pm e_{i} \pm e_{j} ; 1 \leq i \neq j \leq l\right\}
$$

Set

$$
\alpha_{i}=e_{i}-e_{\imath+1}(1 \leq i \leq l-1), \alpha_{l}=2 e_{l} .
$$

In this case Hermitian symmetric spaces are $M\left(\mathfrak{g},\left\{\alpha_{i}\right\}, g\right)(i=1, l)$.

If $\alpha_{i} \in \Psi$ for some $i(2 \leq i \leq l-1)$, then

$$
\alpha=e_{1}+e_{l}=\alpha_{1}+\cdots+\alpha_{l}, \beta=e_{i}-e_{l}=\alpha_{i}+\cdots+\alpha_{l-1}
$$

are elements of $\Delta^{+}(\Psi)$ and satisfy the conditions of Lemma 4.1. Therefore the degree of $M(\mathrm{~g}, \Psi, g)$ is not equal to two.

Let $\Psi=\left\{\alpha_{1}, \alpha_{l}\right\}$. Then set $\alpha=\alpha_{1}$ and $\beta=\alpha_{2}+\cdots+\alpha_{l}$. As above, we see that the degree of $M(\mathrm{~g}, \Psi, g)$ is not equal to two.

The case where $\mathrm{g}$ is of type $D_{l}(l \geq 4)$.

$$
\begin{aligned}
\Delta= & \left\{ \pm e_{i} \pm e_{j} ; 1 \leq i \neq j \leq l\right\} . \\
& \alpha_{i}=e_{i}-e_{i+1}(i=1, \ldots, l-1), \alpha_{l}=e_{l-1}+e_{l} .
\end{aligned}
$$

In this case Hermitian symmetric spaces are $M\left(\mathrm{~g},\left\{\alpha_{i}\right\}, g\right)(i=1, l-1, l)$. 
If $\alpha_{\imath} \in \Psi$ for some $i(2 \leq i \leq l-2)$, then

$$
\alpha=e_{1}-e_{l}=\alpha_{1}+\cdots+\alpha_{l-1}, \beta=e_{\imath}+e_{l}=\alpha_{i}+\cdots+\alpha_{l}
$$

are in $\Delta^{+}(\Psi)$ and satisfy the conditions of Lemma 4.1.

Next we check $M\left(\mathfrak{g},\left\{\alpha_{1}, \alpha_{l}\right\}, g\right)$ and $M\left(\mathfrak{g},\left\{\alpha_{l-1}, \alpha_{l}\right\}, g\right)$.

Set

$$
\alpha=\alpha_{1}+\cdots+\alpha_{l-1}, \beta=\alpha_{2}+\cdots+\alpha_{l-2}+\alpha_{l} .
$$

Then $\alpha$ and $\beta$ satisfy the conditions of Lemma 4.1 and are elements of $\Delta^{+}(\Psi)$, regardless of whether $\Psi=\left\{\alpha_{1}, \alpha_{l}\right\}$ or $\Psi=\left\{\alpha_{l-1}, \alpha_{l}\right\}$.

The case where $\mathrm{g}$ is of type $E_{8}$.

In this case $\Delta$ consists of the following.

$$
\pm e_{i} \pm e_{j}(1 \leq i \neq j \leq 8), \frac{1}{2} \sum_{i=1}^{8} \nu(i) e_{i}\left(\sum \nu(i): \text { even }\right)
$$

Set

$$
\begin{aligned}
& \alpha_{1}=\frac{1}{2}\left(e_{1}+e_{8}-e_{2}-e_{3}-e_{4}-e_{5}-e_{6}-e_{7}\right) \\
& \alpha_{2}=e_{1}+e_{2}, \alpha_{i}=e_{i-1}-e_{i-2}(3 \leq i \leq 8) .
\end{aligned}
$$

We denote a root $\alpha=\sum_{i=1}^{8} n_{i} \alpha_{i}$ by

$$
\left(\begin{array}{lllllll}
n_{8} & n_{7} & n_{6} & n_{5} & n_{4} & n_{3} & n_{1} \\
& & \multicolumn{4}{c}{n_{2}} &
\end{array}\right)
$$

Then there is no $M(\mathfrak{g}, \Psi, g)$ with degree two. In fact, the following $\alpha, \beta$ satisfy the conditions (1) (4) of Lemma 4.1 (cf. [1]).

$$
\alpha=\left(\begin{array}{lllllll}
1 & 1 & 1 & 1 & 1 & 1 & 1 \\
& & & & 1 & &
\end{array}\right), \quad \beta=\left(\begin{array}{lllllll}
1 & 2 & 3 & 4 & 5 & 3 & 1 \\
& & & & 2 & &
\end{array}\right)
$$

The case where $\mathrm{g}$ is of type $E_{7}$.

We use the same notation as in the case $E_{8}$. Then $\left\{\alpha_{1}, \ldots, \alpha_{7}\right\}$ is a fundamental root system and $\Delta$ consists of the following.

$$
\begin{aligned}
& \pm e_{i} \pm e_{j}(1 \leq i \neq j \leq 6), \pm\left(e_{7}-e_{8}\right) \\
& \pm \frac{1}{2}\left(e_{7}-e_{8}+\sum_{i=1}^{6} \nu(i) e_{i}\right)\left(\sum_{i=1}^{6} \nu(i): \text { odd }\right) .
\end{aligned}
$$


In this case Hermitian symmetric space is only $M\left(\mathfrak{g},\left\{\alpha_{7}\right\}, g\right)$. We denote a root $\alpha=\sum_{\imath=1}^{7} n_{i} \alpha_{i}$ by

$$
\left(\begin{array}{llllll}
n_{7} & n_{6} & n_{5} & n_{4} & n_{3} & n_{1} \\
& & & n_{2} & &
\end{array}\right)
$$

Then

$$
\alpha=\left(\begin{array}{llllll}
0 & 1 & 1 & 2 & 1 & 1 \\
& & & 1 & &
\end{array}\right), \quad \beta=\left(\begin{array}{llllll}
1 & 1 & 2 & 2 & 2 & 1 \\
& & & 1 & &
\end{array}\right)
$$

satisfy $(1) \sim(4)$ of Lemma 4.1 .

The case where $\mathrm{g}$ is of type $E_{6}$.

$\Delta$ consists of

$$
\begin{aligned}
& \pm e_{i} \pm e_{j}(1 \leq i \neq j \leq 5) \\
& \pm \frac{1}{2}\left(e_{8}-e_{7}-e_{6}+\sum_{i=1}^{5} \nu(i) e_{i}\right)\left(\sum_{i=1}^{5} \nu(i): \text { even }\right)
\end{aligned}
$$

In this case Hermitian symmetric spaces are $M\left(\mathrm{~g},\left\{\alpha_{i}\right\}, g\right)(i=1,6)$. We identify $\alpha=\sum_{i=1}^{6} n_{i} \alpha_{i}$ with

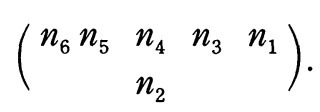

Then

$$
\alpha=\left(\begin{array}{lllll}
0 & 1 & 2 & 1 & 1 \\
& & 1 & &
\end{array}\right), \quad \beta=\left(\begin{array}{lllll}
1 & 1 & 1 & 1 & 0 \\
& & 1 & &
\end{array}\right)
$$

satisfy (1) $\sim(4)$ of Lemma 4.1 .

The case where $\mathrm{g}$ is of type $F_{4}$.

$$
\begin{aligned}
& \Delta=\left\{ \pm e_{i}, \pm e_{i} \pm e_{j}(1 \leq i \neq j \leq 4), \frac{1}{2}\left( \pm e_{1} \pm e_{2} \pm e_{3} \pm e_{4}\right)\right\} \\
& \alpha_{1}=e_{2}-e_{3}, \alpha_{2}=e_{3}-e_{4}, \alpha_{3}=e_{4}, \alpha_{4}=\frac{1}{2}\left(e_{1}-e_{2}-e_{3}-e_{4}\right) .
\end{aligned}
$$

We identify $\alpha=\sum_{i=1}^{4} n_{i} \alpha_{i}$ with $\left(n_{1}, n_{2}, n_{3}, n_{4}\right)$.

If $\Psi$ contains $\alpha_{i}$ for some $i(1 \leq i \leq 3)$, then

$$
\alpha=(1,1,2,2) \text { and } \beta=(1,2,2,0)
$$


are elements of $\Delta^{+}(\Psi)$ and satisfy (1) (4) of Lemma 4.1 .

Let $\Psi=\left\{\alpha_{4}\right\}, \alpha=(0,0,0,1)$ and $\beta=(1,2,3,1)$. Then the degree of $M\left(\mathfrak{g},\left\{\alpha_{4}\right\}, g\right)$ is not equal to two.

The case where $\mathrm{g}$ is of type $G_{2}$.

$\Delta$ consists of the following.

$$
\begin{aligned}
& \pm\left(e_{2}-e_{3}\right), \pm\left(e_{3}-e_{1}\right), \pm\left(e_{1}-e_{2}\right) \\
& \pm\left(2 e_{1}-e_{2}-e_{3}\right), \pm\left(2 e_{2}-e_{1}-e_{3}\right), \pm\left(2 e_{3}-e_{1}-e_{2}\right) .
\end{aligned}
$$

Let $\alpha_{1}=e_{1}-e_{2}$ and $\alpha_{2}=-2 e_{1}+e_{2}+e_{3}$. Then $M\left(\mathfrak{g},\left\{\alpha_{1}\right\}, g\right)$ is a Hermitian symmetric space.

Suppose that $\alpha_{2} \in \Psi$. Then $\alpha=3 \alpha_{1}+\alpha_{2}$ and $\beta=\alpha_{2}$ is contained in $\Delta^{+}(\Psi)$ and satisfy $(1) \sim(4)$.

Finally we check $M\left(B_{2},\{\alpha, \beta\}, g\right)\left(\alpha=e_{1}-e_{2}, \beta=e_{2}\right)$.

We compute $\left(\nabla^{2} R\right)(\alpha, \overline{\alpha+\beta}, \beta ; \alpha, \beta)$. Since

$$
\alpha+\beta, \alpha+2 \beta \in \Delta \quad \text { and } \quad \alpha-\beta, 2 \alpha+\beta \notin \Delta,
$$

we have

$$
\begin{aligned}
( & \left.\nabla^{2} R\right)(\alpha, \overline{\alpha+\beta}, \beta ; \alpha, \beta) \\
= & -\Lambda(\alpha) R(\Lambda(\beta) \alpha, \overline{\alpha+\beta}) \beta-\Lambda(\alpha) R(\alpha, \Lambda(\beta) \overline{\alpha+\beta}) \beta-\Lambda(\beta) R(\alpha, \Lambda(\alpha) \overline{\alpha+\beta}) \beta \\
& +R(\Lambda(\beta) \alpha, \Lambda(\alpha) \overline{\alpha+\beta}) \beta-\Lambda(\beta) R(\alpha, \overline{\alpha+\beta}) \Lambda(\alpha) \beta+R(\Lambda(\beta) \alpha, \overline{\alpha+\beta}) \Lambda(\alpha) \beta \\
& +R(\alpha, \Lambda(\beta) \overline{\alpha+\beta}) \Lambda(\alpha) \beta+R(\alpha, \overline{\alpha+\beta}) \Lambda(\Lambda(\beta) \alpha) \beta .
\end{aligned}
$$

Comparing the above equation with the right hand side of (4.2), we get

$$
\begin{aligned}
& \left(\nabla^{2} R\right)(\alpha, \overline{\alpha+\beta}, \beta ; \alpha, \beta) \\
& =R(\alpha, \overline{\alpha+\beta}) \Lambda(\Lambda(\beta) \alpha) \beta+\Lambda(\alpha) \Lambda(\overline{\alpha+\beta}) \Lambda(\Lambda(\beta) \alpha) \beta-\Lambda(\Lambda(\alpha) \overline{\alpha+\beta}) \Lambda(\Lambda(\beta) \alpha) \beta \\
& \quad+\text { the right hand side of }(4.2) .
\end{aligned}
$$

Thus

$$
\begin{aligned}
& \left(\nabla^{2} R\right)(\alpha, \overline{\alpha+\beta}, \beta ; \alpha, \beta) \\
& =-2 \frac{(c \cdot p(\alpha))(c \cdot p(\beta))^{2}}{(c \cdot p(\alpha+\beta))^{2}(c \cdot p(2 \beta+\alpha))}\left(N_{\alpha, \beta}\right)^{2}\left(N_{\beta, \alpha+\beta}\right)^{2} \cdot(\alpha+\beta) \\
& \quad+\text { the right hand side of }(4.2) .
\end{aligned}
$$


From (4.4), we have

$$
\left(N_{\alpha, \beta}\right)^{2}=\left(N_{\beta, \alpha+\beta}\right)^{2}=e, \alpha\left(H_{\beta}\right)=-e,
$$

where $e=\beta\left(H_{\beta}\right)=(1 / 2) \alpha\left(H_{\alpha}\right)$. Therefore

$$
\begin{aligned}
\left(\nabla^{2} R\right)(\alpha, \overline{\alpha+\beta}, \beta ; \alpha, \beta) \\
=-\frac{c \cdot p(\beta)}{c \cdot p(\alpha+\beta)}\left(N_{\alpha, \beta}\right)^{2}\left\{-\frac{2 e(c \cdot p(\alpha))(c \cdot p(\beta))}{(c \cdot p(\alpha+\beta))(c \cdot p(\alpha+2 \beta))}+\frac{2 e(c \cdot p(\beta))}{c \cdot p(\alpha+\beta)}\right. \\
\left.\quad-2 e-\frac{3 e(c \cdot p(\alpha))}{c \cdot p(\alpha+\beta)}+\frac{e(c \cdot p(\alpha))}{c \cdot p(\alpha+\beta)}\right\} \cdot(\alpha+\beta) \\
=-2 e^{2} \frac{(c \cdot p(\beta))(c \cdot p(\beta))}{(c \cdot p(\alpha+\beta))^{2}(c \cdot p(2 \beta+\alpha))}(c \cdot p(\alpha)+4 c \cdot p(\beta)) \cdot(\alpha+\beta) .
\end{aligned}
$$

Therefore the degree of $M\left(B_{2},\{\alpha, \beta\}, g\right)$ is not equal to two.

We have thus proved the theorem.

\section{Degree three}

For $\alpha_{i} \in \Pi$, set $\Delta_{i}^{+}(k)=\left\{\alpha=\sum_{j} n_{j} \alpha_{j} \in \Delta^{+} ; n_{i}=k\right\}$.

We devote this section to proving the following theorem.

THEOREM 5.1. Let $\alpha_{i}, \alpha_{q}$ and $\alpha_{r}$ be elements of $\Pi$ such that $\Delta_{i}^{+}(k)=\emptyset$, $\Delta_{g}{ }^{+}(m)=\emptyset$ and $\Delta_{r}{ }^{+}(n)=\emptyset$ for $k \geq 3, m, n \geq 2$. Then Kähler $C$-space with degree three is one of $M\left(\mathfrak{g},\left\{\alpha_{i}\right\}, g\right)$ and $M\left(\mathfrak{g},\left\{\alpha_{q}, \alpha_{r}\right\}, g\right)$

At first we show that the degrees of $M\left(\mathfrak{g},\left\{\alpha_{i}\right\}, g\right)$ and $M\left(\mathfrak{g},\left\{\alpha_{q}, \alpha_{r}\right\}, g\right)$ are at most three.

In the following we suppose that $\alpha, \beta, \gamma, \delta, \omega$ and $\lambda$ are elements of $\Delta^{+}(\Psi)$.

Suppose $\Psi=\left\{\alpha_{i}\right\}$. Since

$$
\Lambda\left(\mathfrak{p}^{\mathbf{C}}\right) \mathfrak{p}^{ \pm} \subset \mathfrak{p}^{ \pm}, \quad R\left(\mathfrak{p}^{\mathbf{C}}, \mathfrak{p}^{\mathbf{C}}\right) \mathfrak{p}^{ \pm} \subset \mathfrak{p}^{ \pm},
$$

we can see

$$
\begin{aligned}
& \left(\nabla^{3} R\right)(\alpha, \bar{\lambda}, \beta ; \gamma, \delta, \omega) \in \mathfrak{p}^{+} \\
& \left(\nabla^{3} R\right)(\bar{\alpha}, \lambda, \bar{\beta} ; \gamma, \delta, \omega) \in \mathfrak{p}^{-} .
\end{aligned}
$$

Therefore, If $\left(\nabla^{3} R\right)(\alpha, \bar{\lambda}, \beta ; \gamma, \delta, \omega) \neq 0$, then $\alpha+\beta+\gamma+\delta+\omega-\lambda$ must be in $\Delta^{+}(\Psi)$. Similarly, if $\left(\nabla^{3} R\right)(\bar{\alpha}, \lambda, \bar{\beta} ; \gamma, \delta, \omega) \neq 0$, then $\alpha+\beta-\gamma-\delta-\omega$ $-\lambda$ must be in $\Delta^{+}(\Psi)$. 
Each $\alpha \in \Delta^{+}(\Psi)$ has $1 \leq p(\alpha) \leq 2$ so that

$$
p(\alpha+\beta+\gamma+\delta+\omega-\lambda) \geq 1+1+1+1+1-2=3 .
$$

However, this is impossible, since $\Delta_{i}^{+}(k)=\emptyset$ for $k \geq 3$. Similarly we have

$$
p(\alpha+\beta-\gamma-\delta-\omega-\lambda) \leq 2+2-1-1-1-1=0 .
$$

Thus the degree of $M\left(\mathfrak{g},\left\{\alpha_{1}\right\}, g\right)$ is not more than three.

Next, suppose $\Psi=\left\{\alpha_{q}, \alpha_{r}\right\}(q<r)$. Since ${\Delta_{q}}^{+}(m)=\emptyset$ and $\Delta_{r}{ }^{+}(n)=\emptyset$ for $m, n \geq 2$, it is easy to see that the possibilities of $p(\alpha)$ are only $(1,0),(0,1)$ and $(1,1)$. Therefore

$$
\begin{aligned}
& p(\alpha+\beta+\gamma+\delta+\omega-\lambda) \neq(1,0),(0,1),(1,1) \\
& p(\alpha+\beta-\gamma-\delta-\omega-\lambda) \neq(1,0),(0,1),(1,1) .
\end{aligned}
$$

Thus the degree of $M\left(\mathfrak{g},\left\{\alpha_{q}, \alpha_{r}\right\}, g\right)$ is not more than three.

Next, we prove that Hermitian symmetric spaces, $M\left(\mathfrak{g},\left\{\alpha_{\imath}\right\}, g\right)$ and $M(\mathfrak{g}$, $\left\{\alpha_{q}, \alpha_{r}\right\}, g$ ) are only Kähler $C$-spaces of which degrees are at most three.

As in Section 4, we shall prove the following lemmas.

Lemma 5.2. Suppose that there are $\alpha, \beta, \gamma \in \Delta^{+}(\Psi)(\alpha \neq \beta, \beta \neq \gamma, \gamma \neq \alpha)$ satisfying the following:
(1) $\alpha+\beta \in \Delta$,
(2) $\alpha+\gamma \in \Delta$
(3) $\alpha+\beta+\gamma \in \Delta$,
(4) $\alpha-\beta \notin \Delta$,
( 5 ) $\beta+\gamma \notin \Delta$,
( 6 ) $\beta-\gamma \notin \Delta, \quad$ ( 7 ) $2 \alpha+\beta \notin \Delta$
( 8 ) $2 \beta+\alpha \notin \Delta$,
( 9 ) $2 \alpha+\gamma \notin \Delta$,
(10) $\alpha+\gamma-\beta \notin \Delta$
(11) $2 \alpha+\beta+\gamma \notin \Delta$, (12) $2 \beta+\alpha+\gamma \notin \Delta$,
(13) $2 \alpha+2 \beta+\gamma \notin \Delta$
(14) $\alpha-\gamma \notin \Delta$,
(15) $2 \gamma+\alpha \notin \Delta$.

Then the degree of $M(\mathfrak{g}, \Psi, g)$ is more than three.

Lemma 5.3. Let $\alpha$ and $\beta$ be in $\Delta^{+}(\Psi)(\alpha \neq \beta)$. If the following conditions are satisfied, then the degree of $M(\mathrm{~g}, \Psi, \mathrm{g})$ is more than three:
(1) $\alpha+\beta \in \Delta$,
(2) $\alpha-\beta \notin \Delta$,
(3) $2 \alpha+\beta \notin \Delta$
(4) $2 \beta+\alpha \in \Delta$,
(5) $3 \beta+\alpha \notin \Delta$.

Proof of Lemma 5.2. We shall show

$$
\left(\nabla^{3} R\right)(\alpha, \bar{\lambda}, \beta ; \alpha, \beta, \gamma) \neq 0 \quad(\lambda=\alpha+\beta+\gamma) .
$$

By Theorem 3.4 and (10) of Lemma 5.2, we have 


$$
\begin{aligned}
& \left(\nabla^{3} R\right)(\alpha, \bar{\lambda}, \beta ; \alpha, \beta, \gamma) \\
= & -\left(\Lambda^{2} R\right)(\Lambda(\gamma) \alpha, \bar{\lambda}, \beta ; \alpha, \beta) \\
& -\left(\Lambda^{2} R\right)(\alpha, \Lambda(\gamma) \bar{\lambda}, \beta ; \alpha, \beta) \\
& -\left(\Lambda^{2} R\right)(\alpha, \bar{\lambda}, \beta ; \Lambda(\gamma) \alpha, \beta) .
\end{aligned}
$$

By (4.1) and the conditions of the lemma, we have

$$
\begin{aligned}
( & \left.\nabla^{2} R\right)(\Lambda(\gamma) \alpha, \bar{\lambda}, \beta ; \alpha, \beta) \\
= & -\Lambda(\alpha) R(\Lambda(\beta) \Lambda(\gamma) \alpha, \bar{\lambda}) \beta-\Lambda(\alpha) R(\Lambda(\gamma) \alpha, \Lambda(\beta) \bar{\lambda}) \beta+R(\Lambda(\gamma) \alpha, \Lambda(\Lambda(\beta) \alpha) \bar{\lambda}) \beta \\
& +R(\Lambda(\gamma) \alpha, \Lambda(\alpha) \Lambda(\beta) \bar{\lambda}) \beta-\Lambda(\beta) R(\Lambda(\gamma) \alpha, \bar{\lambda}) \Lambda(\alpha) \beta \\
& +R(\Lambda(\beta) \Lambda(\gamma) \alpha, \bar{\lambda}) \Lambda(\alpha) \beta+R(\Lambda(\gamma) \alpha, \Lambda(\beta) \bar{\lambda}) \Lambda(\alpha) \beta \\
= & \Lambda(\alpha)[[\Lambda(\beta) \Lambda(\gamma) \alpha, \bar{\lambda}], \beta] \\
& +\Lambda(\alpha)\{\Lambda(\Lambda(\beta) \bar{\lambda}) \Lambda(\Lambda(\gamma) \alpha) \beta+[[\Lambda(\gamma) \alpha, \Lambda(\beta) \bar{\lambda}]\} \\
& -\{\Lambda(\Lambda(\Lambda(\beta) \alpha) \bar{\lambda}) \Lambda(\Lambda(\gamma) \alpha) \beta+\Lambda([\Lambda(\gamma) \alpha, \Lambda(\Lambda(\beta) \alpha) \bar{\lambda}] \beta\} \\
& -\{\Lambda(\Lambda(\alpha) \Lambda(\beta) \bar{\lambda}) \Lambda(\Lambda(\gamma) \alpha) \beta+\Lambda([\Lambda(\gamma) \alpha, \Lambda(\alpha) \Lambda(\beta) \bar{\lambda}] \beta\} \\
& +\Lambda(\beta) \Lambda([\Lambda(\gamma) \alpha, \bar{\lambda}]) \Lambda(\alpha) \beta-[[\Lambda(\beta) \Lambda(\gamma) \alpha, \bar{\lambda}], \Lambda(\alpha) \beta] \\
& -[[\Lambda(\gamma) \alpha, \Lambda(\beta) \bar{\lambda}], \Lambda(\alpha) \beta] .
\end{aligned}
$$

Now, put $c_{\alpha}=c \cdot p(\alpha)\left(\alpha \in \Delta^{+}\left(\Psi^{\dagger}\right)\right)$. Then, by Lemma 1.1 and (1.7), we have

$$
\begin{aligned}
&\left(\nabla^{2} R\right)(\Lambda(\gamma) \alpha, \bar{\lambda}, \beta ; \alpha, \beta) \\
&=-\frac{c_{\alpha} c_{\beta} c_{\alpha+\gamma}}{c_{\alpha+\beta} c_{\alpha+\gamma} c_{\lambda}} N_{\gamma, \alpha} N_{\beta,-\lambda} \beta\left(H_{\lambda}\right) \cdot[\alpha, \beta] \\
&+\frac{c_{\alpha} c_{\beta}}{c_{\alpha+\beta} c_{\alpha+\gamma}} N_{\gamma, \alpha} N_{\beta,-\lambda}\left\{\frac{c_{\beta}}{c_{\lambda}}\left(N_{\beta,-\lambda}\right)^{2}+\beta\left(H_{\gamma+\alpha}\right)\right\}[\alpha, \beta] \\
&-\frac{\left(c_{\alpha}\right)^{2} c_{\beta}}{c_{\alpha+\beta} c_{\alpha+\gamma}}\left\{\frac{1}{c_{\alpha+\beta}}\left(N_{r, \alpha}\right)^{2} N_{\beta, \alpha} N_{\gamma,-\lambda}-\frac{1}{c_{\lambda}} N_{\gamma, \alpha} N_{\beta,-\lambda}\left(N_{r,-\lambda}\right)^{2}\right\}[\alpha, \beta] \\
&+\frac{c_{\alpha} c_{\beta}}{c_{\alpha+\gamma}}\left\{\frac{1}{c_{\gamma}}\left(N_{\gamma, \alpha} N_{\beta,-\gamma}\right)^{2} N_{r,-\lambda} \cdot(\alpha+\beta)+\frac{1}{c_{\alpha+\beta}}\left(N_{\gamma, \alpha}\right)^{3} N_{\beta,-\lambda} \cdot[\alpha, \beta]\right\} \\
&-\frac{\left(c_{\alpha}\right)^{2} c_{\beta}}{\left(c_{\alpha+\beta}\right)^{2} c_{\alpha+\gamma}} N_{\gamma, \alpha} N_{\beta,-\lambda}\left(N_{\alpha, \beta}\right)^{2} \cdot[\alpha, \beta]+\frac{c_{\alpha} c_{\beta} c_{\alpha+\gamma}}{c_{\alpha+\beta} c_{\alpha+\gamma} c_{\lambda}} N_{\gamma, \alpha} N_{\beta,-\lambda} \lambda\left(H_{\alpha+\beta}\right) \cdot[\alpha, \beta] \\
&-\frac{c_{\alpha} c_{\beta}}{c_{\alpha+\beta} c_{\alpha+\gamma}} N_{r, \alpha} N_{\beta,-\gamma}(\alpha+\beta)\left(H_{\gamma+\alpha}\right) \cdot[\alpha, \beta] .
\end{aligned}
$$

For simplicity, put $e=\alpha\left(H_{\alpha}\right)$. Then, by (1.9) and the conditions of the lemma, we get the following. 


$$
\begin{aligned}
& \beta\left(H_{\beta}\right)=\gamma\left(H_{r}\right)=e, \alpha\left(H_{\beta}\right)=\alpha\left(H_{r}\right)=-\frac{e}{2} \\
& \beta\left(H_{\gamma}\right)=0,\left(N_{\alpha, \beta}\right)^{2}=\left(N_{\alpha, \gamma}\right)^{2}=\frac{e}{2} .
\end{aligned}
$$

Moreover it follows from (1.8) that

$$
N_{\alpha, \beta} N_{\gamma,-\lambda}+N_{\gamma, \alpha} N_{\beta,-\lambda}=0 .
$$

Therefore (5.1) gives

$$
\left(\Lambda^{2} R\right)(\Lambda(\gamma) \alpha, \bar{\lambda}, \beta ; \alpha, \beta)=\frac{e^{2} N_{\gamma,-\lambda}\left(c_{\alpha}\right)^{2} c_{\beta}}{2\left(c_{\alpha+\beta}\right)^{2} c_{\alpha+\gamma}} \cdot(\alpha+\beta) .
$$

Similarly, we have

$$
\left(\Lambda^{2} R\right)(\alpha, \bar{\lambda}, \beta ; \Lambda(\gamma) \alpha, \beta)=\frac{e^{2} c_{\alpha} c_{\beta}}{2\left(c_{\alpha+\beta}\right)^{2}} N_{\gamma,-\lambda} \cdot(\alpha+\beta) .
$$

From (4.3) we get

$$
\begin{aligned}
& \left(\Lambda^{2} R\right)(\alpha, \Lambda(\gamma) \bar{\lambda}, \beta ; \alpha, \beta) \\
& =N_{\gamma,-\lambda}\left(\Lambda^{2} R\right)(\alpha, \overline{\alpha+\beta}, \beta ; \alpha, \beta) \\
& =-\frac{e^{2} c_{\alpha} c_{\beta}}{\left(c_{\alpha+\beta}\right)^{2}} N_{\gamma,-\lambda} \cdot(\alpha+\beta) .
\end{aligned}
$$

Therefore it follows from (5.2), (5.3) and (5.4) that

$$
\begin{aligned}
& \left(\nabla^{3} R\right)(\alpha, \bar{\lambda}, \beta ; \alpha, \beta, \gamma) \\
& =\frac{e^{2} c_{\alpha} c_{\beta}}{2\left(c_{\alpha+\beta}\right)^{2}} N_{\gamma,-\lambda} \cdot\left\{\frac{c_{\alpha}}{c_{\alpha+\gamma}}+1-2\right\} \cdot(\alpha+\beta) \\
& =-\frac{e^{2} c_{\alpha} c_{\beta} c_{\gamma}}{2\left(c_{\alpha+\beta}\right)^{2} c_{\alpha+\gamma}} N_{\gamma,-\lambda} \cdot(\alpha+\beta) .
\end{aligned}
$$

This completes the proof of Lemma 5.2.

Proof of Lemma 5.3. We shall show that

$$
\left(\Lambda^{3} R\right)(\alpha, \bar{\lambda}, \alpha ; \beta, \beta, \beta) \neq 0(\lambda=2 \beta+\alpha) .
$$

In fact

$$
\begin{aligned}
& \left(\Lambda^{3} R\right)(\alpha, \bar{\lambda}, \alpha ; \beta, \beta, \beta) \\
& =\Lambda(\beta)\left(\Lambda^{2} R\right)(\alpha, \bar{\lambda}, \alpha ; \beta, \beta)
\end{aligned}
$$




$$
\begin{aligned}
& \left(\Lambda^{2} R\right)(\Lambda(\beta) \alpha, \bar{\lambda}, \alpha ; \beta, \beta) \\
& -\left(\Lambda^{2} R\right)(\alpha, \Lambda(\beta) \bar{\lambda}, \alpha ; \beta, \beta) \\
& -\left(\Lambda^{2} R\right)(\alpha, \bar{\lambda}, \Lambda(\beta) \alpha ; \beta, \beta) \\
= & 3 \Lambda(\beta)\{R(\Lambda(\beta) \Lambda(\beta) \alpha, \bar{\lambda}) \alpha+R(\alpha, \Lambda(\beta) \Lambda(\beta) \bar{\lambda}) \alpha \\
& +R(\alpha, \bar{\lambda}) \Lambda(\beta) \Lambda(\beta) \alpha+2 R(\Lambda(\beta) \alpha, \Lambda(\beta) \bar{\lambda}) \alpha \\
& +2 R(\Lambda(\beta) \alpha, \bar{\lambda}) \Lambda(\beta) \alpha+2 R(\alpha, \Lambda(\beta) \bar{\lambda}) \Lambda(\beta) \alpha\} \\
& -3\{R(\Lambda(\beta) \Lambda(\beta) \alpha, \Lambda(\beta) \bar{\lambda}) \alpha+R(\Lambda(\beta) \Lambda(\beta) \alpha, \bar{\lambda}) \Lambda(\beta) \alpha \\
& +R(\Lambda(\beta) \alpha, \Lambda(\beta) \Lambda(\beta) \bar{\lambda}) \alpha+R(\alpha, \Lambda(\beta) \Lambda(\beta) \bar{\lambda}) \Lambda(\beta) \alpha \\
& +R(\Lambda(\beta) \alpha, \bar{\lambda}) \Lambda(\beta) \Lambda(\beta) \alpha+R(\alpha, \Lambda(\beta) \bar{\lambda}) \Lambda(\beta) \Lambda(\beta) \alpha\} \\
& -6 R(\Lambda(\beta) \alpha, \Lambda(\beta) \bar{\lambda}) \Lambda(\beta) \alpha .
\end{aligned}
$$

As before, we set $e=\alpha\left(H_{\alpha}\right)$. Then we obtain

$$
\beta\left(H_{\beta}\right)=\left(N_{\alpha, \beta}\right)^{2}=\left(N_{\beta,-\lambda}\right)^{2}=\frac{e}{2}, \quad \alpha\left(H_{\beta}\right)=-\frac{e}{2} .
$$

Thus, by a straightforward computation we have

$$
\left(\Lambda^{3} R\right)(\alpha, \bar{\lambda}, \alpha ; \beta, \beta, \beta)=\frac{3 e^{2} c_{\alpha}\left(c_{\beta}\right)^{2}}{2\left(c_{\alpha+\beta}\right)^{3}} N_{\beta,-\lambda} \cdot(\alpha+\beta) .
$$

We have thus proved the lemma.

Suppose that $g$ is not of $G_{2}$ type. For Kähler $C$-spaces except for those stated in Theorem 5.1, we take examples of $\{\alpha, \beta, \gamma\}$ satisfying the conditions of Lemma 5.2 or of $\{\alpha, \beta\}$ satisfying the conditions of Lemma 5.3.

The case where $\mathrm{g}$ is of type $A_{l}(l \geq 3)$.

Suppose that $\alpha_{i}, \alpha_{j}$ and $\alpha_{k}$ are elements of $\Psi(i<j<k)$. Then set

$$
\alpha=\alpha_{1}+\cdots+\alpha_{j-1}, \beta=\alpha_{j}, \gamma=\alpha_{j+1}+\cdots+\alpha_{l} .
$$

Then $\alpha, \beta$ and $\gamma$ satisfy (1) (15) of Lemma 5.2.

The case where $\mathrm{g}$ is of type $B_{l}(l \geq 2)$.

We use the notation in Section 4 .

Suppose that $\Psi$ contains $\alpha_{i}$ and $\alpha_{j}(i<j)$. Put

$$
\alpha=\alpha_{i}=e_{i}-e_{i+1}, \beta=e_{i+1}=\alpha_{i+1}+\cdots+\alpha_{l} .
$$

Then $\alpha$ and $\beta$ satisfy (1) (5) of Lemma 5.3. 
The case where $\mathrm{g}$ is of type $C_{l}(l \geq 3)$.

Suppose that $\Psi$ contains $\alpha_{i}$ and $\alpha_{j}(i<j)$. Put $\beta=\alpha_{i}+\cdots+\alpha_{j-1}=e_{i}-$ $e_{j}$ and

$$
\alpha=2 e_{j}= \begin{cases}\alpha_{l} & \text { if } j=l, \\ 2 \alpha_{j}+\cdots+2 \alpha_{l-1}+\alpha_{l} & \text { if } j<l .\end{cases}
$$

Then $\alpha$ and $\beta$ satisfy (1) (5) of Lemma 5.3.

The case where $\mathrm{g}$ is of type $D_{l}(l \geq 4)$.

Suppose that $\Psi$ contains $\left\{\alpha_{i}, \alpha_{l}\right\}(2 \leq i \leq l-2)$. Then put

$$
\alpha=\alpha_{l}=e_{l-1}+e_{l}, \beta=\alpha_{2}+\cdots+\alpha_{l-1}=e_{2}-e_{l}, \gamma=\alpha_{1}+\cdots+\alpha_{l-2}=e_{1}-e_{l-1} \text {. }
$$

Then $\alpha, \beta$ and $\gamma$ are contained in $\Delta^{+}(\Psi)$ and satisfy (1) (15) in Lemma 5.2.

Next, we assume that $\Psi$ cotains $\left\{\alpha_{i}, \alpha_{j}\right\}(1 \leq i<j \leq l-2)$. Set

$$
\alpha=\alpha_{1}+\cdots+\alpha_{j-1}, \beta=\alpha_{j}+\cdots+\alpha_{l-2}+\alpha_{l-1}, \gamma=\alpha_{j}+\cdots+\alpha_{l-2}+\alpha_{l} .
$$

Then $\alpha, \beta$ and $\gamma$ are contained in $\Delta^{+}(\Psi)$ and satisfy (1) (15) in Lemma 5.2.

The case where $\mathrm{g}$ is of type $E_{8}$.

Set

$$
\begin{aligned}
& \alpha=\left(\begin{array}{lllllll}
0 & 1 & 1 & 2 & 2 & 1 & 1 \\
& & & & 1 & &
\end{array}\right), \quad \beta=\left(\begin{array}{lllllll}
1 & 1 & 1 & 1 & 2 & 1 & 0 \\
& & & & 1 & &
\end{array}\right) \text {, } \\
& \gamma=\left(\begin{array}{lllllll}
1 & 1 & 2 & 2 & 2 & 2 & 1 \\
& & & & 1 & &
\end{array}\right)
\end{aligned}
$$

Then $\alpha, \beta$ and $\gamma$ satisfy (1) (15) in Lemma 5.2 .

The case where $\mathrm{g}$ is of type $E_{7}$.

Put

$$
\begin{aligned}
& \alpha=\left(\begin{array}{llllll}
1 & 1 & 1 & 1 & 1 & 0 \\
& & & 0 & &
\end{array}\right), \quad \beta=\left(\begin{array}{llllll}
0 & 1 & 1 & 1 & 1 & 1 \\
& & & 1 & &
\end{array}\right), \\
& \gamma=\left(\begin{array}{llllll}
0 & 0 & 1 & 2 & 1 & 1 \\
& & & 1 & &
\end{array}\right) .
\end{aligned}
$$

Then $\alpha, \beta$ and $\gamma$ satisfy (1) (15) in Lemma 5.2. Therefore, if $\Psi$ contains $\alpha_{i}(i=$ $3,4$ or 5$)$, the degree of $M(\mathrm{~g}, \Psi, g)$ is more than three. Moreover, if $\Psi$ contains $\left\{\alpha_{1}, \alpha_{6}\right\},\left\{\alpha_{1}, \alpha_{7}\right\},\left\{\alpha_{2}, \alpha_{6}\right\}$ or $\left\{\alpha_{2}, \alpha_{7}\right\}$, the degree of $M(\mathfrak{g}, \Psi, g)$ is more than 
three.

Next, set

$$
\begin{gathered}
\alpha=\left(\begin{array}{llllll}
0 & 1 & 1 & 1 & 1 & 0 \\
& & & 1 & &
\end{array}\right), \quad \beta=\left(\begin{array}{llllll}
1 & 1 & 1 & 1 & 1 & 1 \\
& & & 0 & &
\end{array}\right), \\
\gamma=\left(\begin{array}{llllll}
0 & 0 & 1 & 2 & 1 & 1
\end{array}\right) .
\end{gathered}
$$

Then the degree of $M\left(\mathrm{~g},\left\{\alpha_{1}, \alpha_{2}\right\}, g\right)$ is more than three.

Finally, suppose that $\Psi=\left\{\alpha_{6}, \alpha_{7}\right\}$. Set

$$
\begin{aligned}
& \alpha=\left(\begin{array}{llllll}
1 & 0 & 0 & 0 & 0 & 0 \\
& & & 0 & &
\end{array}\right), \quad \beta=\left(\begin{array}{llllll}
0 & 1 & 1 & 1 & 1 & 1 \\
& & & 1 & &
\end{array}\right), \\
& \gamma=\left(\begin{array}{llllll}
0 & 1 & 3 & 3 & 2 & 1 \\
& & & 1 & &
\end{array}\right) \text {. }
\end{aligned}
$$

Then $\alpha, \beta$ and $\gamma$ are contained in $\Delta^{+}(\Psi)$ and satisfy (1) (15) in Lemma 5.2.

The case where $\mathrm{g}$ is of type $E_{6}$.

Set

$$
\begin{gathered}
\alpha=\left(\begin{array}{ccccc}
1 & 1 & 1 & 0 & 0 \\
& & 0 &
\end{array}\right), \quad \beta=\left(\begin{array}{lllll}
0 & 0 & 1 & 1 & 1 \\
& & 1 &
\end{array}\right), \\
\gamma=\left(\begin{array}{lllll}
0 & 1 & 1 & 1 & 0 \\
& & 1 &
\end{array}\right) .
\end{gathered}
$$

Thus we can see that the degree of $M(\mathfrak{g}, \Psi, g)$ is more than three if $\Psi$ contains one of the following:

$$
\left\{\alpha_{4}\right\},\left\{\alpha_{2}, \alpha_{5}\right\},\left\{\alpha_{2}, \alpha_{6}\right\},\left\{\alpha_{3}, \alpha_{5}\right\},\left\{\alpha_{3}, \alpha_{6}\right\} .
$$

Finally, we check the case where $\Psi=\left\{\alpha_{5}, \alpha_{6}\right\}$. Then the following roots $\alpha, \beta$ and $\gamma$ are contained in $\Delta^{+}(\Psi)$ and satisfy the conditions in Lemma 5.2:

$$
\begin{aligned}
& \alpha=\left(\begin{array}{lllll}
0 & 1 & 0 & 0 & 0 \\
& & 0 & &
\end{array}\right), \quad \beta=\left(\begin{array}{lllll}
0 & 1 & 1 & 1 & 1 \\
& & 1 & &
\end{array}\right), \\
& r=\left(\begin{array}{lllll}
0 & 1 & 2 & 1 & 0 \\
& & 1 & &
\end{array}\right) .
\end{aligned}
$$

The case where $\mathrm{g}$ is of type $F_{4}$.

Set $\alpha=(1,1,2,2)$ and $\beta=(0,1,1,0)$. Then $\alpha$ and $\beta$ satisfy (1) $\sim(5)$ of Lemma 5.3. Thus, if $\alpha_{i} \in \Delta^{+}(\Psi)(i=2$ or 3$)$, than the degree of $M(\mathrm{~g}, \Psi, g)$ is more 
than three.

Next, let $\Psi=\left\{\alpha_{1}, \alpha_{4}\right\}$. Then put $\alpha=(1,1,0,0)$ and $\beta=(0,0,1,1)$. Then $\alpha$ and $\beta$ satisfy (1) $\sim(5)$ of Lemma 5.3 .

Finally we shall prove that the degree of $M\left(G_{2},\left\{\alpha_{1}, \alpha_{2}\right\}, g\right)$ is more than three. Set $\alpha=\alpha_{2}$ and $\beta=\alpha_{1}$. Then $\Delta^{+}$consists of the following:

$$
\alpha, \beta, \alpha+\beta, \alpha+2 \beta, \alpha+3 \beta, 2 \alpha+3 \beta .
$$

Therefore we have from (1.9)

$$
\begin{aligned}
& \left(N_{\alpha, \beta}\right)^{2}=\frac{3}{2} \beta\left(H_{\beta}\right),\left(H_{\alpha+\beta, \beta}\right)^{2}=2 \beta\left(H_{\beta}\right), \\
& \left(N_{-\beta, \alpha+3 \beta}\right)^{2}=\frac{3}{2} \beta\left(H_{\beta}\right), \alpha\left(H_{\alpha}\right)=3 \beta\left(H_{\beta}\right), \alpha\left(H_{\beta}\right)=-\frac{3}{2} \beta\left(H_{\beta}\right) .
\end{aligned}
$$

We show that

$$
\left(\nabla^{3} R\right)(\alpha, \overline{\alpha+3 \beta}, \beta ; \beta, \beta, \beta) \neq 0 .
$$

From Theorem 3.4 we have

$$
\begin{aligned}
( & \left.\nabla^{3} R\right)(\alpha, \overline{\alpha+3 \beta}, \beta ; \beta, \beta, \beta) \\
= & -\left(\Lambda^{2} R\right)(\Lambda(\beta) \alpha, \overline{\alpha+3 \beta}, \beta ; \beta, \beta) \\
& -\left(\Lambda^{2} R\right)(\alpha, \Lambda(\beta) \overline{\alpha+3 \beta}, \beta ; \beta, \beta) \\
= & -3\{R(\Lambda(\beta) \alpha, \Lambda(\beta) \Lambda(\beta) \overline{\alpha+3 \beta}) \beta+R(\Lambda(\beta) \Lambda(\beta) \alpha, \Lambda(\beta) \overline{\alpha+3 \beta}) \beta\} \\
& -R(\alpha, \Lambda(\beta) \Lambda(\beta) \Lambda(\beta) \overline{\alpha+3 \beta}) \beta-R(\Lambda(\beta) \Lambda(\beta) \Lambda(\beta) \alpha, \overline{\alpha+3 \beta}) \beta \\
= & N_{\beta, \alpha} N_{-\beta, \alpha+3 \beta} N_{\beta, \alpha+\beta}\left\{3 \frac{c_{\alpha}}{c_{\alpha+2 \beta}}\left(\frac{c_{\beta}}{c_{\alpha+2 \beta}}\left(N_{\beta, \alpha+\beta}\right)^{2}+\beta\left(H_{\alpha+\beta}\right)\right)\right. \\
& -3 \frac{c_{\alpha}}{c_{\alpha+2 \beta}}\left(\frac{c_{\beta}}{c_{\alpha+3 \beta}}\left(N_{-\beta, \alpha+3 \beta}\right)^{2}+\beta\left(H_{\alpha+2 \beta}\right)\right) \\
& \left.-\left(\frac{c_{\beta}}{c_{\alpha+\beta}}\left(N_{\alpha, \beta}\right)^{2}+\beta\left(H_{\alpha}\right)\right)+\frac{c_{\alpha}}{c_{\alpha+3 \beta}} \beta\left(H_{\alpha+3 \beta}\right)\right\} \cdot \beta \\
= & \frac{12 c_{a}\left(c_{\beta}\right)^{2}}{c_{\alpha+\beta} c_{\alpha+2 \beta} c_{\alpha+3 \beta}} N_{\beta, \alpha} N_{-\beta, \alpha+3 \beta} N_{\beta, \alpha+\beta} \beta\left(H_{\beta}\right) \cdot \beta \\
\neq & 0 .
\end{aligned}
$$

Therefore the degree of $M\left(G_{2},\{\alpha, \beta\}, g\right)$ is more than three.

We have thus proved Theorem 5.1. 


\section{REFERENCES}

[1] Hans Freudenthal and H. de Vries, Linear Lie Groups, Academic Press, 1969.

[2] S. Helgason, Differential Geometry, Lie Groups, and Symmetric Spaces, Academic Press, 1978.

[3] M. Itoh, On curvature properties of Kähler $C$-spaces, J. Math. Soc. Japan, 30 (1976), 39-71.

[4] S. Kobayashi and K. Nomizu, Foundations of Differential Geometry II, Interscience Publishers, 1969.

[ 5 ] K. Nomizu, Invariant affine connections on homogeneous spaces, Amer. J. Math., 76 (1954), 33-65.

[6] R. Takagi, On higher covariant derivatives of the curvature tensors of Kählerian C-spaces, Nagoya Math. J., 91 (1983), 1-18.

[ 7 ] J. A. Wolf, On the classification of hermitian symmetric spaces, J. Math. Mech., 13 (1964), 489-495.

Graduate School of Science and Technology

Chiba University

Chiba-shi, 263 Japan 